\title{
The Pseudo-Linear Semantics of Interval-Valued Fuzzy Logics
}

\author{
B. Van Gasse ${ }^{1}$, C. Cornelis $^{1}$, G. Deschrijver, E. E. Kerre \\ Fuzziness and Uncertainty Modelling Research Unit \\ Department of Applied Mathematics and Computer Science \\ Ghent University, Krijgslaan 281 (S9), 9000 Gent, Belgium
}

\begin{abstract}
Triangle algebras are equationally defined structures that are equivalent with certain residuated lattices on a set of intervals, which are called interval-valued residuated lattices (IVRLs). Triangle algebras have been used to construct Triangle Logic (TL), a formal fuzzy logic that is sound and complete w.r.t. the class of IVRLs.

In this paper, we prove that the so-called pseudo-prelinear triangle algebras are subdirect products of pseudo-linear triangle algebras. This can be compared with MTL-algebras (prelinear residuated lattices) being subdirect products of linear residuated lattices.

As a consequence, we are able to prove the pseudo-chain completeness of Pseudolinear Triangle Logic (PTL), an axiomatic extension of TL introduced in this paper. This kind of completeness is the analogue of the chain completeness of MTL (Monoidal T-norm based Logic).

This result also provides a better insight in the structure of triangle algebras; it enables us, amongst others, to prove properties of pseudo-prelinear triangle algebras more easily. It is known that there is a one-to-one correspondence between triangle algebras and couples $(\mathcal{L}, \alpha)$, in which $\mathcal{L}$ is a residuated lattice and $\alpha$ an element in that residuated lattice. We give a schematic overview of these properties (and a number of others that can be imposed on a triangle algebra), and the corresponding necessary and sufficient conditions on $\mathcal{L}$ and $\alpha$.
\end{abstract}

Key words:

interval-valued fuzzy set theory, residuated lattices, formal logic

1991 MSC: 03B45, 03B47, 03B50, 03B52, 06F05, 08A72

Email addresses: Bart.VanGasse@UGent. be (B. Van Gasse), Chris.Cornelis@UGent. be (C. Cornelis), Glad.Deschrijver@UGent. be

(G. Deschrijver), Etienne.Kerre@UGent.be (E. E. Kerre).

1 Bart Van Gasse and Chris Cornelis would like to thank the Research FoundationFlanders for funding their research. 


\section{Introduction and preliminaries}

One of the most general formal fuzzy logics is Höhle's Monoidal Logic (ML) [16]. This is a Hilbert-style deduction system, with axiom schemes and deduction rules. ML is sound and complete w.r.t. the variety of residuated lattices ${ }^{2}$ [6], which are structures $\mathcal{L}=(L, \sqcap, \sqcup, *, \Rightarrow, 0,1)$ in which $\sqcap, \sqcup, *$ and $\Rightarrow$ are binary operators on the set $L$ and

- $(L, \sqcap, \sqcup)$ is a bounded lattice with 0 as smallest and 1 as greatest element,

- * is commutative and associative, with 1 as neutral element, and

- $x * y \leq z$ iff $x \leq y \Rightarrow z$ for all $x, y$ and $z$ in $L$ (residuation principle).

ML is the basis for a number of well-known stronger formal fuzzy logics, such as Esteva and Godo's Monoidal T-norm based Logic (MTL) [8], Hájek's Basic Logic (BL) [14], Łukasiewicz Logic (LL) [20], Intuitionistic Logic (IL) [15] and Gödel Logic (GL) [12]. These logics are sound and complete w.r.t. MTLalgebras, BL-algebras, MV-algebras (or, equivalently, Wajsberg algebras [10]), Heyting-algebras and G-algebras, respectively. Below, we recall the definitions of these concepts, along with some other important notions. We refer to [9] and [13] for a comprehensive overview of these and other logics.

Definition 1 We will use the notations $\neg x$ for $x \Rightarrow 0, x \Leftrightarrow y$ for $(x \Rightarrow$ $y) \sqcap(y \Rightarrow x)$ and $x^{n}$ for $\underbrace{x * x * \ldots * x}_{n \text { times }}$. By definition, $x^{0}=1$.

- An MTL-algebra [8] is a prelinear residuated lattice, i.e., a residuated lattice in which $(x \Rightarrow y) \sqcup(y \Rightarrow x)=1$ for all $x$ and $y$ in $L$.

- A BL-algebra [14] is a divisible MTL-algebra, i.e., an MTL-algebra in which $x \sqcap y=x *(x \Rightarrow y)$ for all $x$ and $y$ in $L$. The weaker property $x \sqcap y=$ $(x *(x \Rightarrow y)) \sqcup(y *(y \Rightarrow x))$ is called weak divisibility [26,27].

- An MV-algebra [1,2] is a BL-algebra in which the negation is an involution, i.e., $(x \Rightarrow 0) \Rightarrow 0=x$ for all $x$ in $L$.

- A Heyting-algebra, or pseudo-Boolean algebra [23], is a residuated lattice in which $x * x=x$ for all $x$ in $L$, or, equivalently, in which $x * y=x \sqcap y$ for all $x$ and $y$ in $L$.

$\overline{2}$ In literature (e.g. in [16]), the name residuated lattice is sometimes used for more general structures than what we call residuated lattices. In the most general terminology, our structures would be called bounded integral commutative residuated lattices. 
- A G-algebra [14] is a prelinear Heyting-algebra.

- A Boolean algebra [17] is an MV-algebra that is also a Heyting-algebra.

If a residuated lattice satisfies $x \sqcup y=((x \Rightarrow y) \Rightarrow y) \sqcap((y \Rightarrow x) \Rightarrow x)$, for all $x$ and $y$ in $L$, then it is called $\sqcup$-definable [8,9]. The stronger property $x \sqcup y=(x \Rightarrow y) \Rightarrow y$ is called strong $\sqcup$-definability ${ }^{3}$ [26,27].

In general, these algebraic structures are not linearly ordered. So the general semantics of ML, MTL, BL, GL, LL, etc. are not linear. For MTL, and stronger logics (such as BL, LL and GL) however, it is possible to restrict ourselves to linear semantics. Indeed, these logics are not only sound and complete w.r.t. the corresponding subvarieties (of the variety of residuated lattices), but even w.r.t. the linear structures only [8]. For example, MTL is sound and complete w.r.t. linear MTL-algebras, BL is sound and complete w.r.t. linear BL-algebras, and so on. The reason is that prelinearity holds in MTL-algebras (and therefore also in BL-algebras, MV-algebras, etc.) Indeed, because of this property, it can be proven that MTL-algebras are isomorphic to subdirect products of linear MTL-algebras, that BL-algebras are isomorphic to subdirect products of linear BL-algebras, and so on. Therefore each of these varieties is generated by its linearly ordered structures.

Moreover, in some cases the structures on the unit interval $([0,1]$, min, max $)$ suffice to generate the complete variety. For example, the variety of MTLalgebras is generated by the MTL-algebras on $([0,1], \min , \max )[18]$, which are called standard MTL-algebras. Similarly, the variety of BL-algebras is generated by the BL-algebras on $([0,1], \min , \max )[3]$, which are called standard BL-algebras. So for the logics MTL and BL, we do not only have general and linear semantics, but even standard semantics. This is also true for LL and GL [14]. It should however be noted that stronger logics do not automatically inherit the property 'having standard semantics' (in contrast with the property 'having linear semantics') [9]. The standard MTL-algebras and BLalgebras are exactly those that are induced by left-continuous and continuous t-norms, respectively. Therefore MTL is sometimes referred to as the logic of left-continuous t-norms, and BL as the logic of continuous t-norms.

Definition 2 A triangular norm (t-norm, for short) [24] on a bounded lattice $(L, \sqcap, \sqcup, 0,1)$ is a binary, increasing, commutative and associative operator $T: L^{2} \rightarrow L$ that satisfies $T(x, 1)=1$, for all $x$ in $L$.

If for every pair $(x, y)$ in $L^{2}, \sup \{z \in L \mid T(x, z) \leq y\}$ exists, then the map $I_{T}$ defined by $I_{T}(x, y)=\sup \{z \in L: T(x, z) \leq y\}$ is called the residual implicator of $T$.

$\overline{3}$ Strong $\sqcup$-definable residuated lattices are exactly MV-algebras [16]. 
In a residuated lattice, the operator $*$ is always a t-norm, and $\Rightarrow$ is its residual implicator.

In [27], we introduced Triangle Logic (TL). This is basically Monoidal Logic (ML) [16] enriched with three connectives, representing 'uncertainty', 'necessity' and 'possibility', respectively: $\bar{u}, \square$ and $\diamond$. So the language of TL consists of countably many proposition variables $\left(p_{1}, p_{2}, \ldots\right)$, the constants $\overline{0}$ and $\bar{u}$, the unary operators $\square, \diamond$, the binary operators $\wedge, \vee, \&, \rightarrow$, and finally the auxiliary symbols '(' and ')'. Formulas are defined inductively: proposition variables, $\overline{0}$ and $\bar{u}$ are formulas; if $\phi$ and $\psi$ are formulas, then so are $(\phi \wedge \psi)$, $(\phi \vee \psi),(\phi \& \psi),(\phi \rightarrow \psi),(\square \phi)$ and $(\diamond \phi)$.

The following notations are used: $\overline{1}$ for $\overline{0} \rightarrow \overline{0}, \neg \phi$ for $\phi \rightarrow \overline{0}$ and $\phi \leftrightarrow \psi$ for $(\phi \rightarrow \psi) \wedge(\psi \rightarrow \phi)$, for formulas $\phi$ and $\psi$.

The axioms of TL are those of ML, i.e.,

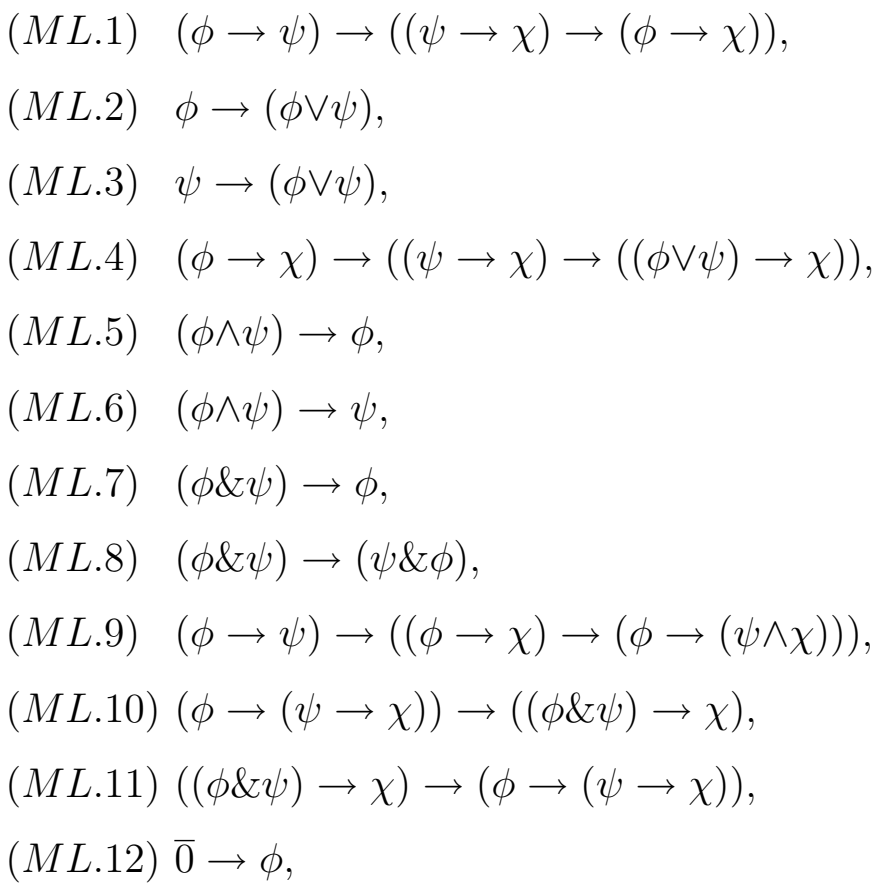


complemented with ${ }^{4}$

$$
\begin{array}{ll}
(T L .1) \square \phi \rightarrow \phi, & \left(T L .1^{\prime}\right) \phi \rightarrow \diamond \phi, \\
(T L .2) \square \phi \rightarrow \square \square \phi, & \left(T L .2^{\prime}\right) \diamond \diamond \phi \rightarrow \diamond \phi, \\
(T L .3)(\square \phi \wedge \square \psi) \rightarrow \square(\phi \wedge \psi), & \left(T L .3^{\prime}\right)(\diamond \phi \wedge \diamond \psi) \rightarrow \diamond(\phi \wedge \psi), \\
(T L .4) \square(\phi \vee \psi) \rightarrow(\square \phi \vee \square \psi), & \left(T L .4^{\prime}\right) \diamond(\phi \vee \psi) \rightarrow(\diamond \phi \vee \diamond \psi), \\
(T L .5) \neg \square \bar{u}, & \left(T L .5^{\prime}\right) \diamond \bar{u}, \\
(T L .6) \diamond \phi \rightarrow \square \diamond \phi, & \left(T L .6^{\prime}\right) \diamond \square \phi \rightarrow \square \phi, \\
(T L .7) \square(\phi \rightarrow \psi) \rightarrow(\square \phi \rightarrow \square \psi), & \\
(T L .8)(\square \phi \leftrightarrow \square \psi) \&(\diamond \phi \leftrightarrow \diamond \psi) \rightarrow(\phi \leftrightarrow \psi), & \\
(T L .9)(\square \phi \rightarrow \square \psi) \rightarrow \square(\square \phi \rightarrow \square \psi) . &
\end{array}
$$

The deduction rules are modus ponens (MP, from $\phi$ and $\phi \rightarrow \psi$ infer $\psi$ ), generalization ( $\mathrm{G}$, from $\phi$ infer $\square \phi)$ ) and monotonicity of $\diamond(\mathrm{M} \diamond$, from $\phi \rightarrow \psi$ infer $\diamond \phi \rightarrow \diamond \psi)$. Theories, provability, evaluations and models are defined in the usual way. Triangle Logic is sound and complete w.r.t. interval-valued residuated lattices (IVRLs), and axiomatic extensions of this expansion of ML are sound and complete w.r.t. the corresponding subclasses of the class of IVRLs. This was proven in [27]. The following definitions are also from [27].

Definition 3 Given a lattice $\mathcal{L}=(L, \sqcap, \sqcup)$, its triangularization $\mathbb{T}(\mathcal{L})$ is the structure $\mathbb{T}(\mathcal{L})=(\operatorname{Int}(\mathcal{L}), \sqcap, \sqcup)$ defined by

- $\operatorname{Int}(\mathcal{L})=\left\{\left[x_{1}, x_{2}\right] \mid\left(x_{1}, x_{2}\right) \in L^{2}\right.$ and $\left.x_{1} \leq x_{2}\right\}$,

- $\left[x_{1}, x_{2}\right] \sqcap\left[y_{1}, y_{2}\right]=\left[x_{1} \sqcap y_{1}, x_{2} \sqcap y_{2}\right]$,

- $\left[x_{1}, x_{2}\right] \sqcup\left[y_{1}, y_{2}\right]=\left[x_{1} \sqcup y_{1}, x_{2} \sqcup y_{2}\right]$.

The set $D_{\mathcal{L}}=\{[x, x] \mid x \in L\}$ is called the diagonal of $\mathbb{T}(\mathcal{L})$.

The triangularization of $([0,1]$, min, max $)$ is denoted as $\mathcal{L}^{I}=\left(L^{I}, \sqcap, \sqcup\right)$.

Definition 4 An interval-valued residuated lattice (IVRL) is a residuated lattice $\left(\operatorname{Int}(\mathcal{L}), \sqcap, \sqcup, \odot, \Rightarrow_{\odot},[0,0],[1,1]\right)$ on the triangularization $\mathbb{T}(\mathcal{L})$ of a bounded lattice $\mathcal{L}$, in which the diagonal $D_{\mathcal{L}}$ is closed under $\odot$ and $\Rightarrow_{\odot}$,

4 The definition of TL in [27] included two additional axioms: $\overline{1}$ and $\neg \diamond \overline{0}$. However, they can be proven from the remaining axioms and hence are omitted here.Indeed, for $\square \overline{1}$ we already noted this in [27]; for $\neg \diamond \overline{0}$, this follows from the fact that the following formulas are provably equivalent: $\diamond \overline{0}, \diamond \square \bar{u}, \square \bar{u}$ and $\overline{0}$. 
i.e., $[x, x] \odot[y, y] \in D_{\mathcal{L}}$ and $[x, x] \Rightarrow_{\odot}[y, y] \in D_{\mathcal{L}}$ for all $x, y$ in $L$.

So TL is a logic which has interval-valued structures as its (general) semantics. The idea behind interval-valued truth degrees is that they provide a way to express incomplete as well as graded knowledge (see e.g. [4,7,11,21,22,26,27]). In fact, interval-valued fuzzy sets are a special case of type-2 fuzzy sets, which were introduced in [29].

Example 5 [5] If $T$ is a left-continuous t-norm on ([0,1], min, $\max ), \alpha \in$ $[0,1]$ and the mapping $\mathcal{T}_{T, \alpha}$ is defined, for $x=\left[x_{1}, x_{2}\right]$ and $y=\left[y_{1}, y_{2}\right]$ in $L^{I}$, by the formula

$$
\mathcal{T}_{T, \alpha}(x, y)=\left[T\left(x_{1}, y_{1}\right), \max \left(T\left(\alpha, T\left(x_{2}, y_{2}\right)\right), T\left(x_{1}, y_{2}\right), T\left(x_{2}, y_{1}\right)\right)\right],
$$

then $\left(L^{I}, \sqcap, \sqcup, \mathcal{I}_{T, \alpha}, \mathcal{I}_{\mathcal{T}_{T, \alpha}},[0,0],[1,1]\right)$ is an IVRL, in which $\mathcal{I}_{\mathcal{T}_{T, \alpha}}$ is the residual implicator of $\mathcal{T}_{T, \alpha}$ :

$$
\mathcal{I}_{\mathcal{T}_{T, \alpha}}(x, y)=\left[\min \left(I_{T}\left(x_{1}, y_{1}\right), I_{T}\left(x_{2}, y_{2}\right)\right), \min \left(I_{T}\left(T\left(x_{2}, \alpha\right), y_{2}\right), I_{T}\left(x_{1}, y_{2}\right)\right)\right] .
$$

In [27], we introduced the notion of triangle algebra, a structure that serves as an equational representation for an interval-valued residuated lattice. Triangle algebras form the link between IVRLs and TL.

Definition 6 A triangle algebra is a structure $\mathcal{A}=(A, \sqcap, \sqcup, *, \Rightarrow, \nu, \mu, 0, u, 1)$, in which $(A, \sqcap, \sqcup, *, \Rightarrow, 0,1)$ is a residuated lattice, $\nu$ and $\mu$ are unary operators, $u$ a constant, and satisfying the following conditions:

$$
\begin{array}{ll}
(T .1) \nu x \leq x, & \left(T .1^{\prime}\right) x \leq \mu x, \\
(T .2) \nu x \leq \nu \nu x, & \left(T .2^{\prime}\right) \mu \mu x \leq \mu x, \\
(T .3) \nu(x \sqcap y)=\nu x \sqcap \nu y, & \left(T .3^{\prime}\right) \mu(x \sqcap y)=\mu x \sqcap \mu y, \\
(T .4) \nu(x \sqcup y)=\nu x \sqcup \nu y, & \left(T .4^{\prime}\right) \mu(x \sqcup y)=\mu x \sqcup \mu y, \\
(T .5) \nu u=0, & \left(T .5^{\prime}\right) \mu u=1, \\
(T .6) \nu \mu x=\mu x, & \left(T .6^{\prime}\right) \mu \nu x=\nu x, \\
(T .7) \nu(x \Rightarrow y) \leq \nu x \Rightarrow \nu y, & \\
(T .8)(\nu x \Leftrightarrow \nu y) *(\mu x \Leftrightarrow \mu y) \leq(x \Leftrightarrow y), & \\
(T .9) \nu x \Rightarrow \nu y \leq \nu(\nu x \Rightarrow \nu y) . &
\end{array}
$$

In [27], we established a one-to-one correspondence between IVRLs and triangle algebras. It is shown in Figure 1. The unary operators $\nu$ and $\mu$ correspond 


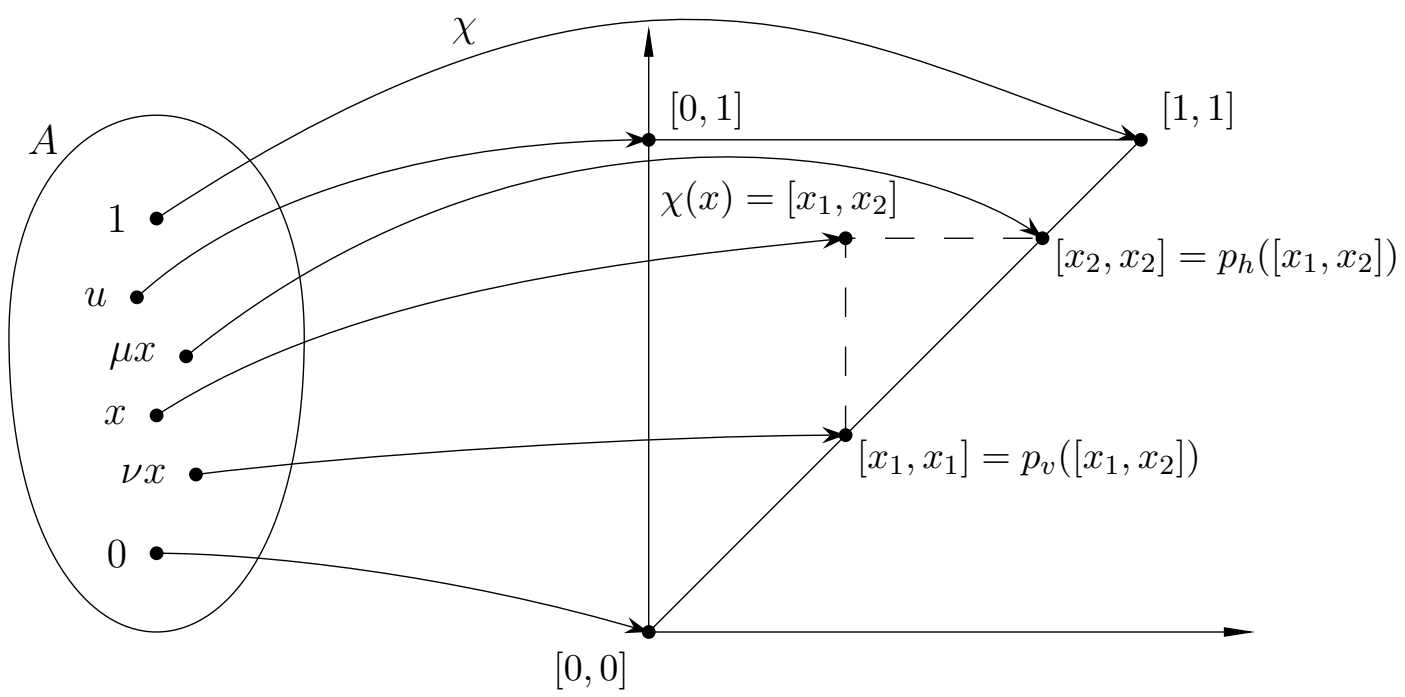

Triangle algebra

Isomorphic triangle algebra $(A, \sqcap, \sqcup, *, \Rightarrow, \nu, \mu, 0, u, 1)$

$\left(A^{\prime}, \sqcap^{\prime}, \sqcup^{\prime}, *^{\prime}, \Rightarrow^{\prime}, p_{v}, p_{h},[0,0],[0,1],[1,1]\right)$ in which $\left(A^{\prime}, \sqcap^{\prime}, \sqcup^{\prime}, *^{\prime}, \Rightarrow^{\prime},[0,0],[1,1]\right)$ is an IVRL

Fig. 1. The isomorphism $\chi$ from a triangle algebra to an IVRL.

with the mappings that map $\left[x_{1}, x_{2}\right]$ to $\left[x_{1}, x_{1}\right]$ and $\left[x_{2}, x_{2}\right]$ respectively. The constant $u$ corresponds to $[0,1]$. Theorem 7 gives this connection in more detail:

Theorem 7 [27] There is a one-to-one correspondence between the class of IVRLs and the class of triangle algebras. Every extended IVRL ${ }^{5}$ is a triangle algebra and conversely, every triangle algebra is isomorphic to an extended IVRL.

Definition 8 [27] Let $\mathcal{A}=(A, \sqcap, \sqcup, *, \Rightarrow, \nu, \mu, 0, u, 1)$ be a triangle algebra. An element $x$ in $A$ is called exact if $\nu x=x$. The set of exact elements of $\mathcal{A}$ is denoted by $E(\mathcal{A})$.

It was proven in [27] that $E(\mathcal{A})$ is closed under all the defined operations on $\mathcal{A}$. Every property in Definition 1 (prelinearity, divisibility, ...) can therefore be weakened, by imposing it on $E(\mathcal{A})$ (instead of $A$ ) only. We will denote this with the prefix 'pseudo'. For example, a triangle algebra is said to be pseudo-linear if its set of exact elements is linearly ordered (by the original (restricted) ordering). Another example: a triangle algebra is pseudo-divisible if $\nu x \sqcap \nu y=\nu x *(\nu x \Rightarrow \nu y)$ for all $x$ and $y$ in $A(E(\mathcal{A})$ consists exactly of the

5 An extended IVRL is simply an IVRL in which the two mentioned projections are defined and the constant $[0,1]$ is fixed. 
elements of the form $\nu x$ ).

In [28] we proved that

Theorem 9 In a triangle algebra $\mathcal{A}=(A, \sqcap, \sqcup, *, \Rightarrow, \nu, \mu, 0, u, 1)$, the implication $\Rightarrow$ and the product $*$ are completely determined by their action on $E(\mathcal{A})$ and the value of $u * u$. More specifically:

- $\nu(x \Rightarrow y)=(\nu x \Rightarrow \nu y) \sqcap(\mu x \Rightarrow \mu y)$,

- $\mu(x \Rightarrow y)=(\mu x \Rightarrow(\mu(u * u) \Rightarrow \mu y)) \sqcap(\nu x \Rightarrow \mu y)$,

- $\nu(x * y)=\nu x * \nu y$

- $\mu(x * y)=(\nu x * \mu y) \sqcup(\mu x * \nu y) \sqcup(\mu x * \mu y * \mu(u * u))$.

For any $x$ in a triangle algebra, it holds that $x=\nu x \sqcup(\mu x \sqcap u)$ (see [28]). Therefore, $x$ is completely determined by $\nu x$ and $\mu x$ (which are elements of $E(\mathcal{A}))$ : if $\nu x=\nu y$ and $\mu x=\mu y$, then $x=y$.

In this paper, we introduce the concept of filters of triangle algebras and use it to prove that pseudo-prelinear triangle algebras are subdirect products of pseudo-linear triangle algebras (Section 2). This is analogous to the property that prelinear residuated lattices are subdirect products of linear residuated lattices [8]. That result was used to show that MTL is chain complete. In a similar way, we obtain that Pseudo-linear Triangle Logic (PTL), which is TL extended with the axiom scheme $(\square \phi \rightarrow \square \psi) \vee(\square \psi \rightarrow \square \phi)$ (pseudoprelinearity), is pseudo-chain complete, i.e., complete w.r.t. pseudo-linear triangle algebras. In other words, PTL has pseudo-linear semantics.

Because of Theorem 9, the product and implication in triangle algebras are always of a specific form, which implies that a triangle algebra is completely determined by its subalgebra of exact elements and the value $u * u$. Conversely, for a fixed residuated lattice $\mathcal{L}$ and element $\alpha$ in that lattice, we can construct a triangle algebra with $\mathcal{L}$ as subalgebra of exact elements and $u * u$ determined by $\mu(u * u)=\alpha$. In other words, there is a one-to-one correspondence between triangle algebras and couples $(\mathcal{L}, \alpha)$, in which $\alpha$ is an element in the residuated lattice $\mathcal{L}$. This characterization implies that every property that can be imposed on triangle algebras, can be formulated in terms of such couples. In Section 3, for some interesting properties, we are able to find such necessary and sufficient conditions. We present them in a table at the end of this paper. 


\section{Filters and completeness results}

Now we introduce the concept of filters of triangle algebras and apply this to prove that certain triangle algebras are subdirect products of triangle algebras with a linear diagonal. After that, we give some important consequences of this representation.

Definition 10 Let $\mathcal{A}=(A, \sqcap, \sqcup, *, \Rightarrow, \nu, \mu, 0, u, 1)$ be a triangle algebra. A filter of $\mathcal{A}$ is a non-empty subset $F$ of $A$, satisfying:

(F.1) if $x \in F, y \in A$ and $x \leq y$, then $y \in F$;

(F.2) if $x, y \in F$, then $x * y \in F$;

(F.3) if $x \in F$, then $\nu x \in F$.

Note that (F.1) and (F.2) are the usual conditions for a filter of a residuated lattice.

$A$ filter $F$ of $\mathcal{A}$ is called a prime filter of $\mathcal{A}$ iff for every $x$ and $y$ in $A, \nu x \Rightarrow \nu y$ or $\nu y \Rightarrow \nu x$ is (or both are) in $F$. Or, equivalently, iff for every $x$ and $y$ in $E(\mathcal{A}), x \Rightarrow y$ or $y \Rightarrow x$ is (or both are) in $F$.

For all $x$ and $y$ in $A$, we write $x \sim_{F} y$ iff $x \Rightarrow y$ and $y \Rightarrow x$ are both in $F$.

Because of (F.1), a filter always contains the element 1. Remark that (F.2) and (F.3) can be replaced by "If $x, y \in F$, then $\nu(x * y) \in F$ ".

Proposition 11 Let $F$ be a filter of a triangle algebra $\mathcal{A}=(A, \sqcap, \sqcup, *, \Rightarrow$, $\nu, \mu, 0, u, 1)$. Then the relation $\sim_{F}$ is a congruence relation on $\mathcal{A}$.

PROOF. The fact that $\sim_{F}$ is an equivalence relation, is proven in a similar way as in [14]. Indeed, reflexivity follows from $x \Rightarrow x=1$, for every $x$ in $A$; symmetry is trivial; and transitivity follows from $(x \Rightarrow y) *(y \Rightarrow z) \leq x \Rightarrow z$, for every $x, y$ and $z$ in $A$. Now suppose $x_{1} \sim_{F} x_{2}$ and $y_{1} \sim_{F} y_{2}$. We have to prove that $x_{1} \sqcap y_{1} \sim_{F} x_{2} \sqcap y_{2}, x_{1} \sqcup y_{1} \sim_{F} x_{2} \sqcup y_{2}, x_{1} * y_{1} \sim_{F} x_{2} * y_{2}$, $x_{1} \Rightarrow y_{1} \sim_{F} x_{2} \Rightarrow y_{2}, \nu x_{1} \sim_{F} \nu x_{2}$ and $\mu x_{1} \sim_{F} \mu x_{2}$. Again, the first four claims are proven similarly as in [14]. We now show the remaining two. Because $F$ contains $x_{1} \Rightarrow x_{2}$, it also contains $\nu\left(x_{1} \Rightarrow x_{2}\right)$, which equals $\left(\nu x_{1} \Rightarrow \nu x_{2}\right) \sqcap$ $\left(\mu x_{1} \Rightarrow \mu x_{2}\right)$ by Theorem 9 . Therefore also $\nu x_{1} \Rightarrow \nu x_{2}$ and $\mu x_{1} \Rightarrow \mu x_{2}$ are in $F$. Similarly, also $\nu x_{2} \Rightarrow \nu x_{1}$ and $\mu x_{2} \Rightarrow \mu x_{1}$ are in $F$. So indeed $\nu x_{1} \sim_{F} \nu x_{2}$ and $\mu x_{1} \sim_{F} \mu x_{2}$.

As a corollary, we can meaningfully consider the quotient algebra $\mathcal{A}_{F}=$ $\left(A_{F}, \sqcap_{F}, \sqcup_{F}, *_{F}, \Rightarrow_{F}, \nu_{F}, \mu_{F},[0]_{F},[u]_{F},[1]_{F}\right)$, in which $A_{F}$ is the set of equivalence classes (induced by $\sim_{F}$ ) in $A$, and for any $x$ and $y$ in $A,[x]_{F}=\{y \in$ $\left.A \mid x \sim_{F} y\right\},[x]_{F} \circ_{F}[y]_{F}=[x \circ y]_{F}($ for $\circ \in\{\sqcap, \sqcup, *, \Rightarrow\}), \nu_{F}[x]_{F}=[\nu x]_{F}$ and $\mu_{F}[x]_{F}=[\mu x]_{F}$. This structure is again a triangle algebra, as all defining 
properties are inherited from $\mathcal{A}$. For example, (T.8) follows from

$$
\begin{aligned}
& \left(\left(\nu_{F}[x]_{F} \Leftrightarrow_{F} \nu_{F}[y]_{F}\right) *_{F}\left(\mu_{F}[x]_{F} \Leftrightarrow_{F} \mu_{F}[y]_{F}\right)\right) \sqcup_{F}\left([x]_{F} \Leftrightarrow_{F}[y]_{F}\right) \\
& =[((\nu x \Leftrightarrow \nu y) *(\mu x \Leftrightarrow \mu y)) \sqcup(x \Leftrightarrow y)]_{F} \\
& =[x \Leftrightarrow y]_{F} \\
& =[x]_{F} \Leftrightarrow_{F}[y]_{F} .
\end{aligned}
$$

Proposition 12 Let $\mathcal{A}=(A, \sqcap, \sqcup, *, \Rightarrow, \nu, \mu, 0, u, 1)$ be a triangle algebra and $F$ a filter of $\mathcal{A}$. Then $F$ is a prime filter if and only if $E\left(\mathcal{A}_{F}\right)$ is linearly ordered.

PROOF. First note that for any $x$ and $y$ in $A,[x]_{F} \leq_{F}[y]_{F}$ iff $[x]_{F} \Rightarrow_{F}$ $[y]_{F}=[1]_{F}$ iff $[x \Rightarrow y]_{F}=[1]_{F}$ iff $(x \Rightarrow y) \Rightarrow 1 \in F$ and $1 \Rightarrow(x \Rightarrow y) \in F$ iff $x \Rightarrow y \in F$. Now, every element of $E\left(\mathcal{A}_{F}\right)$ is of the form $\nu_{F}[x]_{F}$ (with $x$ in $A)$, so we have:

$E\left(\mathcal{A}_{F}\right)$ is linearly ordered

- iff for all $x$ and $y$ in $A, \nu_{F}[x]_{F} \leq_{F} \nu_{F}[y]_{F}$ or $\nu_{F}[y]_{F} \leq_{F} \nu_{F}[x]_{F}$

- iff for all $x$ and $y$ in $A,[\nu x]_{F} \leq_{F}[\nu y]_{F}$ or $[\nu y]_{F} \leq_{F}[\nu x]_{F}$

- iff for all $x$ and $y$ in $A, \nu x \Rightarrow \nu y \in F$ or $\nu y \Rightarrow \nu x \in F$

- iff $F$ is a prime filter of $\mathcal{A}$.

Lemma 13 Let $F$ be a filter of a triangle algebra $\mathcal{A}=(A, \sqcap, \sqcup, *, \Rightarrow, \nu, \mu, 0, u, 1)$, and $z \in A$. Then there exists a smallest filter $F_{z}$ containing the elements of $F \cup\{z\}$, and $F_{z}=\left\{v \in A \mid(\exists w \in F)(\exists n \in \mathbb{N})\left(w *(\nu z)^{n} \leq v\right)\right\}$.

\section{PROOF.}

- It is clear that $F_{z}$ contains all elements of $F$, and $z$.

- Every filter that contains $z$, also contains $\nu z, \nu z * \nu z, \nu z * \nu z * \nu z, \ldots$ If such a filter contains an element $w \in F$, it also contains $w * \nu z, w *(\nu z)^{2}, \ldots$ So it is clear that a filter which is a superset of $F \cup\{z\}$, is also a superset of $\left\{v \in A \mid(\exists w \in F)(\exists n \in \mathbb{N})\left(w *(\nu z)^{n} \leq v\right)\right\}$.

- The set $\left\{v \in A \mid(\exists w \in F)(\exists n \in \mathbb{N})\left(w *(\nu z)^{n} \leq v\right)\right\}$ is a filter:

- (F.1) If $v_{1}$ is an element and $v_{1} \leq v_{2}$, then it is clear that $v_{2}$ is an element too (we can take the same $w$ and $n$ ).

- (F.2) If $v_{1}$ and $v_{2}$ are elements of this set, then there exist $w_{1}$ and $w_{2}$ in $F$ and $n_{1}$ and $n_{2}$ in $\mathbb{N}$ such that $w_{1} *(\nu z)^{n_{1}} \leq v_{1}$ and $w_{2} *(\nu z)^{n_{2}} \leq v_{2}$. 


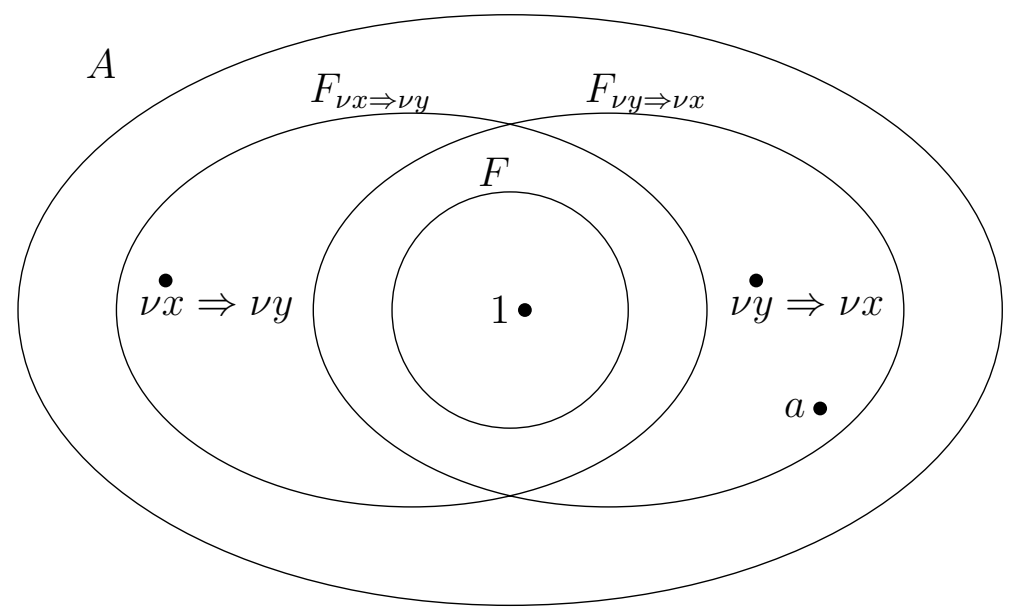

Fig. 2. In this situation, the filter $F_{\nu x \Rightarrow \nu y}$ is a superset of $F \cup\{\nu x \Rightarrow \nu y\}$ not containing $a$.

Therefore $\left(w_{1} * w_{2}\right) *(\nu z)^{n_{1}+n_{2}} \leq v_{1} * v_{2}$. As $w_{1} * w_{2} \in F$ and $n_{1}+n_{2} \in \mathbb{N}$, $v_{1} * v_{2}$ is also an element of the set.

- (F.3) If $w *(\nu z)^{n} \leq v$, then $\nu w *(\nu z)^{n}=\nu\left(w *(\nu z)^{n}\right) \leq \nu v$. So, $\nu v$ is an element if $v$ is, because $\nu w \in F$ if $w \in F$.

Proposition 14 Let $\mathcal{A}$ be a pseudo-prelinear triangle algebra and $a \in A \backslash\{1\}$. Then there exists a prime filter $F$ of $A$ not containing a.

PROOF. First notice that there is a filter of $\mathcal{A}$ not containing $a:\{1\}$. Now suppose $F$ is a filter of $\mathcal{A}$, not containing $a$ and such that, for some $x$ and $y$ in $A$, neither $\nu x \Rightarrow \nu y$ nor $\nu y \Rightarrow \nu x$ are in $F$. We show that there exists a filter $F^{\prime}$, for which $a \notin F^{\prime}, F \subseteq F^{\prime}$ and $\nu x \Rightarrow \nu y \in F^{\prime}$ or $\nu y \Rightarrow \nu x \in F^{\prime}$.

We will do this by showing that $a$ is not in $F_{\nu x \Rightarrow \nu y} \cap F_{\nu y \Rightarrow \nu x}$, as this implies that at least one of the filters $F_{\nu x \Rightarrow \nu y}$ and $F_{\nu y \Rightarrow \nu x}$ satisfies the conditions for $F^{\prime}$. This is illustrated in Figure 2.

If $a$ was in $F_{\nu x \Rightarrow \nu y} \cap F_{\nu y \Rightarrow \nu x}$, then there exist $w_{1}$ and $w_{2}$ in $F$, and $n_{1}$ and $n_{2}$ in $\mathbb{N}$ such that $w_{1} *(\nu(\nu x \Rightarrow \nu y))^{n_{1}} \leq a$ and $w_{2} *(\nu(\nu y \Rightarrow \nu x))^{n_{2}} \leq a$. This 
would imply

$$
\begin{aligned}
& w_{1} * w_{2} \\
& =w_{1} * w_{2} * 1 \\
& =\left(w_{1} * w_{2}\right) *\left((\nu x \Rightarrow \nu y)^{\max \left(n_{1}, n_{2}\right)} \sqcup(\nu y \Rightarrow \nu x)^{\max \left(n_{1}, n_{2}\right)}\right) \\
& =\left(\left(w_{1} * w_{2}\right) *(\nu x \Rightarrow \nu y)^{\max \left(n_{1}, n_{2}\right)}\right) \sqcup\left(\left(w_{1} * w_{2}\right) *(\nu y \Rightarrow \nu x)^{\max \left(n_{1}, n_{2}\right)}\right) \\
& \leq\left(w_{1} *(\nu x \Rightarrow \nu y)^{n_{1}}\right) \sqcup\left(w_{2} *(\nu y \Rightarrow \nu x)^{n_{2}}\right) \\
& \leq a \sqcup a \\
& =a
\end{aligned}
$$

a contradiction because this would mean that $F$ contains $a$. In this calculation we used the properties $(\nu x \Rightarrow \nu y)^{\max \left(n_{1}, n_{2}\right)} \sqcup(\nu y \Rightarrow \nu x)^{\max \left(n_{1}, n_{2}\right)}=1$ (which is valid because $E(\mathcal{A})$ is an MTL-algebra), and $\nu x \Rightarrow \nu y$ is in $E(\mathcal{A})$.

Now, if $A$ is countable, we can number all the pairs of elements of $A$ and construct a sequence of filters $F_{0}=\{1\}, F_{1}, F_{2}, \ldots$ by defining $F_{i+1}=\left(F_{i}\right)_{(\nu x \Rightarrow \nu y)}$ or $F_{i+1}=\left(F_{i}\right)_{(\nu y \Rightarrow \nu x)}$ (the one that does not contain $\left.a\right)$, in which $\{x, y\}$ is the $(i+1)$ th pair in the row. The union of these filters is the desired prime filter not containing $a$.

If $A$ is uncountable, we use Zorn's Lemma: "Every non-empty partially ordered set, in which every chain has an upper bound, contains at least one maximal element." We apply this to the partially ordered set of filters of $\mathcal{A}$ not containing $a$ (with the inclusion as order). Every chain in this set indeed has an upper bound: the union of the filters of the chain is again a filter not containing $a$. It is easy to verify that every maximal element of the set is a prime filter. Indeed; suppose that such a maximal filter $F_{m}$ not containing $a$ does not contain $\nu x \Rightarrow \nu y$, nor $\nu y \Rightarrow \nu x$ for some $x$ and $y$ in $A$. Then, according to our proof, we can construct a filter that is a superset of $F_{m}$ containing $\nu x \Rightarrow \nu y$ or $\nu y \Rightarrow \nu x$, but not $a$. This is a contradiction, as this would mean that $F_{m}$ is not maximal in the set of filters of $\mathcal{A}$ not containing $a$. This concludes the proof.

Exactly as in [14], we can use the previous two propositions to prove

Proposition 15 Every pseudo-prelinear triangle algebra $\mathcal{A}$ is a subalgebra of the direct product of a system of pseudo-linear triangle algebras.

PROOF. Let $\mathcal{F}$ be the system of all prime filters of $\mathcal{A}$. We consider the direct product of the pseudo-linear (because of Proposition 12) triangle algebras $\mathcal{A}^{*}=\left\{\mathcal{A}_{F} \mid F \in \mathcal{F}\right\}$. Now we embed $\mathcal{A}$ into $\mathcal{A}^{*}$, by defining $i(x)$ as $\left\{[x]_{F} \mid F \in\right.$ $\mathcal{F}\}$, for all $x$ in $A$. The mapping $i$ clearly preserves operations. We now show that $i$ is an injection. Suppose $x \neq y$ for $x$ and $y$ in $A$. Then, without loss of generality, we can assume that $x \not \leq y$, which is equivalent with $x \Rightarrow y \neq 1$. Because of Proposition 14, there exists a prime filter $F$ not containing $x \Rightarrow y$. 
This is equivalent with $[x]_{F} \not \leq[y]_{F}$ (see the proof of Proposition 12), which implies $[x]_{F} \neq[y]_{F}$ and therefore $i(x) \neq i(y)$.

This proposition is also valid for subvarieties of pseudo-prelinear triangle algebras. For example, every $\sqcup$-definable pseudo-prelinear triangle algebra is a subalgebra of the direct product of a system of $\sqcup$-definable pseudo-linear triangle algebras. This is due to the fact that the quotient algebras of a $\sqcup$-definable pseudo-prelinear triangle algebra are also $\sqcup$-definable (and similarly for other possible properties), which is proven in an analogous way as we explained after Proposition 11.

Definition 16 Pseudo-linear Triangle Logic (PTL) is the axiomatic extension of Triangle Logic, obtained by adding the axiom scheme $(\square \phi \rightarrow \square \psi) \vee$ $(\square \psi \rightarrow \square \phi)$.

Together with the completeness result in [27] (which is exactly the equivalence of the first two statements in Theorem 17), Proposition 15 implies the following result:

Theorem 17 For each formula $\phi$, the following three statements are equivalent:

- $\phi$ can be deduced from a theory $V$ in PTL,

- for every pseudo-prelinear triangle algebra $\mathcal{A}$ and for every $\mathcal{A}$-model e of $V$, $e(\phi)=1$,

- for every pseudo-linear triangle algebra $\mathcal{A}$ and for every $\mathcal{A}$-model e of $V$, $e(\phi)=1$.

PROOF. The equivalence of the first two statements was proven in [27]. It is also obvious that the second statement implies the third one; we now prove the converse. Suppose that for every pseudo-linear triangle algebra $\mathcal{A}$ and for every $\mathcal{A}$-model $e$ of $V, e(\phi)=1$. Now consider a pseudo-prelinear triangle algebra $\mathcal{A}_{0}$ and an $\mathcal{A}_{0}$-model $e_{0}$ of $V$. Because of Proposition 15 , we know that $\mathcal{A}_{0}$ is a subdirect product of pseudo-linear triangle algebras. The mapping $e_{0}$ can therefore be identified with a system (one for each prime filter $F$ of $\mathcal{A}$ ) of mappings $\left(e_{0}\right)_{F}$ (from the set of formulas into $\mathcal{A}_{F}$ ), defined by $\left(e_{0}\right)_{F}(\chi)=\left[e_{0}(\chi)\right]_{F}$. Each $\mathcal{A}_{F}$ is a pseudo-linear triangle algebra, and each $\left(e_{0}\right)_{F}$ is a $\mathcal{A}_{F}$-model of $V$, so we can use our assumption to conclude that $\left(e_{0}\right)_{F}(\phi)=[1]_{F}$ for each prime filter $F$ in $\mathcal{A}$. This means that $e_{0}(\phi)=1$, exactly what we wanted to prove.

This completeness result remains valid for schematic extensions of PTL. The reason is that Proposition 15 also holds for subvarieties of pseudo-prelinear tri- 
angle algebras. Some examples of axioms that could be added were discussed in $[27]$.

\section{Algebraic properties of triangle algebras}

Another consequence of Proposition 15 is that identities valid in every pseudolinear triangle algebra remain valid in every pseudo-prelinear triangle algebra, because the satisfaction of identities is conserved under direct products. In [26], we proved a number of properties for a class of t-norms on $\mathcal{L}^{I}$. This class consisted exactly of the t-norms described in Formula (1). In the proofs, no properties of $\mathcal{L}^{I}$ are used - apart from the linearity of the diagonal. So - using the one-to-one correspondence between (pseudo-linear) IVRLs and (pseudo-linear) triangle algebras, and Theorem 9 - we can conclude that these properties are even valid in every pseudo-linear triangle algebra. Many of these properties (e.g. all identities) are therefore valid in every pseudoprelinear triangle algebra. We give some examples below. In the calculations, we use, amongst others, Theorem 9.

(1) By straightforward verification - using $x=y$ iff $\nu x=\nu y$ and $\mu x=\mu y$ - one can check that a triangle algebra is distributive iff its diagonal is distributive.

As a linear residuated lattice is always distributive, this implies that distributivity holds in every pseudo-linear triangle algebra. As distributivity is expressed with an identity, we can immediately state that it also holds in every pseudo-prelinear triangle algebra.

(2) Weak divisibility [26] is equivalent with pseudo-divisibility in pseudoprelinear triangle algebras, i.e., every pseudo-prelinear triangle algebra satisfying $x \sqcap y=(x *(x \Rightarrow y)) \sqcup(y *(y \Rightarrow x))$ also satisfies $\nu x \sqcap \nu y=$ $\nu x *(\nu x \Rightarrow \nu y)$, and vice versa. Indeed, every weak divisible pseudoprelinear triangle algebra is a subdirect product of weak divisible pseudolinear triangle algebras (which are known to be pseudo-divisible [26]). As a subdirect product of pseudo-divisible triangle algebras, it is pseudodivisible. The argumentation for the converse is completely analogous. Remark however that the pseudo-prelinearity is in fact not necessary to prove that a weak divisible triangle algebra is pseudo-divisible, as we have 


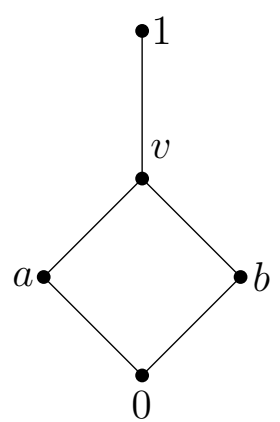

Fig. 3. The Heyting-algebra on this lattice is divisible, but not every IVRL based on it is weak divisible.

$$
\begin{aligned}
\nu x & \sqcap \nu y \\
= & \nu((x \sqcup u) \sqcap \nu y) \\
= & \nu(((x \sqcup u) *((x \sqcup u) \Rightarrow \nu y)) \sqcup(\nu y *(\nu y \Rightarrow(x \sqcup u)))) \\
= & (\nu(x \sqcup u) *((\nu(x \sqcup u) \Rightarrow \nu \nu y) \sqcap(\mu(x \sqcup u) \Rightarrow \mu \nu y))) \sqcup \\
& (\nu \nu y *((\nu \nu y \Rightarrow \nu(x \sqcup u)) \sqcap(\mu \nu y \Rightarrow \mu(x \sqcup u)))) \\
= & (\nu x *((\nu x \Rightarrow \nu y) \sqcap(1 \Rightarrow \nu y))) \sqcup(\nu y *((\nu y \Rightarrow \nu x) \sqcap(\nu y \Rightarrow 1))) \\
= & (\nu x * \nu y) \sqcup(\nu y *(\nu y \Rightarrow \nu x)) \\
= & \nu y *(\nu y \Rightarrow \nu x) .
\end{aligned}
$$

The converse is not true: not every pseudo-divisible triangle algebra is weak divisible. For example, take the Heyting-algebra $\mathcal{L}$ on the lattice ${ }^{6}$ in Figure 3 and consider the IVRL corresponding ${ }^{7}$ to the couple $(\mathcal{L}, \alpha)$, in which $\alpha$ can be $0, a, b$ or $v$ (but not 1 ). Such an IVRL is pseudoHeyting and therefore pseudo-divisible. But it is not weak divisible, because $([a, 1] *([a, 1] \Rightarrow[b, 1])) \sqcup([b, 1] *([b, 1] \Rightarrow[a, 1]))=([a, 1] *[b, 1]) \sqcup$ $([b, 1] *[a, 1])=[0, a \sqcup b \sqcup \alpha]=[0, v \sqcup \alpha]=[0, v]<[0,1]=[a, 1] \sqcap[b, 1]$.

(3) Every pseudo-strong $\sqcup$-definable triangle algebra, i.e., every triangle algebra satisfying $\nu x \sqcup \nu y=(\nu x \Rightarrow \nu y) \Rightarrow \nu y$, is $\sqcup$-definable. Indeed, pseudo-strong $\sqcup$-definability means that the diagonal is an MV-algebra and therefore prelinear. As a pseudo-prelinear, pseudo-strong $\sqcup$-definable triangle algebra, it is a subdirect product of pseudo-linear, pseudo-strong $\sqcup$-definable triangle algebras, which are $\sqcup$-definable [26]. And as a subdirect product of $\sqcup$-definable triangle algebras, it is $\sqcup$-definable.

(4) Every $\sqcup$-definable triangle algebra that satisfies $u * u=u$ (or equivalently

$\overline{6}$ This lattice is also used in [9]. It is example 5.1.20(3) in [19].

7 See the end of Section 1 for details. 
$\mu(u * u)=1$ ), also satisfies pseudo-strong $\sqcup$-definability. Indeed,

$$
\begin{aligned}
\nu x & \sqcup \nu y \\
= & \nu((x \sqcup u) \sqcup \nu y) \\
= & \nu((((x \sqcup u) \Rightarrow \nu y) \Rightarrow \nu y) \sqcap((\nu y \Rightarrow(x \sqcup u)) \Rightarrow(x \sqcup u))) \\
= & (\nu((x \sqcup u) \Rightarrow \nu y) \Rightarrow \nu \nu y) \sqcap(\mu((x \sqcup u) \Rightarrow \nu y) \Rightarrow \mu \nu y) \sqcap \\
& (\nu(\nu y \Rightarrow(x \sqcup u)) \Rightarrow \nu(x \sqcup u)) \sqcap(\mu(\nu y \Rightarrow(x \sqcup u)) \Rightarrow \mu(x \sqcup u)) \\
= & (((\nu(x \sqcup u) \Rightarrow \nu \nu y) \sqcap(\mu(x \sqcup u) \Rightarrow \mu \nu y)) \Rightarrow \nu \nu y) \sqcap \\
& (((\nu(x \sqcup u) \Rightarrow \mu \nu y) \sqcap((\mu(x \sqcup u) * \mu(u * u)) \Rightarrow \mu \nu y)) \Rightarrow \mu \nu y) \sqcap \\
& (((\nu \nu y \Rightarrow \nu(x \sqcup u)) \sqcap(\mu \nu y \Rightarrow \mu(x \sqcup u))) \Rightarrow \nu(x \sqcup u)) \sqcap \\
& (\mu(\nu y \Rightarrow(x \sqcup u)) \Rightarrow 1) \\
= & (((\nu x \Rightarrow \nu y) \sqcap(1 \Rightarrow \nu y)) \Rightarrow \nu y) \sqcap(((\nu x \Rightarrow \nu y) \sqcap((1 * 1) \Rightarrow \nu y)) \Rightarrow \nu y) \sqcap \\
& (((\nu y \Rightarrow \nu x) \sqcap(\nu y \Rightarrow 1)) \Rightarrow \nu x) \sqcap 1 \\
= & (\nu y \Rightarrow \nu y) \sqcap(\nu y \Rightarrow \nu y) \sqcap((\nu y \Rightarrow \nu x) \Rightarrow \nu x) \\
= & (\nu y \Rightarrow \nu x) \Rightarrow \nu x .
\end{aligned}
$$

Remark that it is impossible to prove this if $u * u$ does not equal $u$, even if pseudo-prelinearity is assumed. Indeed, consider the Heyting-algebra with three elements (say, 0, $a$ and 1) and take on its triangularization (see Figure 4$)$ the IVRL determined by $[0,1] *[0,1]=[0,0]$. This IVRL is pseudolinear, but not pseudo-strong $\sqcup$-definable. It is $\sqcup$-definable however. We only need to check this for the two incomparable elements $[0,1]$ and $[a, a]$ (if $x \leq y$, then $((x \Rightarrow y) \Rightarrow y) \sqcap((y \Rightarrow x) \Rightarrow x)=y \sqcap((y \Rightarrow x) \Rightarrow x)=$ $y=x \sqcup y)$. We find $(([0,1] \Rightarrow[a, a]) \Rightarrow[a, a]) \sqcap(([a, a] \Rightarrow[0,1]) \Rightarrow[0,1])=$ $([a, 1] \Rightarrow[a, a]) \sqcap([0,1] \Rightarrow[0,1])=[a, 1] \sqcap[1,1]=[a, 1]=[0,1] \sqcup[a, a]$.

(5) A triangle algebra is a Heyting-algebra iff its diagonal is a Heyting algebra and $u * u=u$. Indeed, in a Heyting-algebra $x * y=x \sqcap y$, for all $x$ and $y$. So $u * u=u \sqcap u=u$. As a subalgebra of a Heyting-algebra, the diagonal is also a Heyting-algebra. Conversely, if the diagonal of a triangle algebra is a Heyting-algebra, and $u * u=u$, then we find, for all $x$ and $y$, that $\nu(x * y)=\nu x * \nu y=\nu x \sqcap \nu y=\nu(x \sqcap y)$ and $\mu(x * y)=$ $(\mu(u * u) * \mu x * \mu y) \sqcup(\nu x * \mu y) \sqcup(\mu x * \nu y)=(\mu x * \mu y) \sqcup(\nu x * \mu y) \sqcup(\mu x * \nu y)=$ $\mu x * \mu y=\mu x \sqcap \mu y=\mu(x \sqcap y)$, which implies that $x * y=x \sqcap y$ (using $(\mathrm{T} .8))$.

Proposition 18 A triangle algebra $\mathcal{A}=(A, \sqcap, \sqcup, *, \Rightarrow, \nu, \mu, 0, u, 1)$ is prelin- 


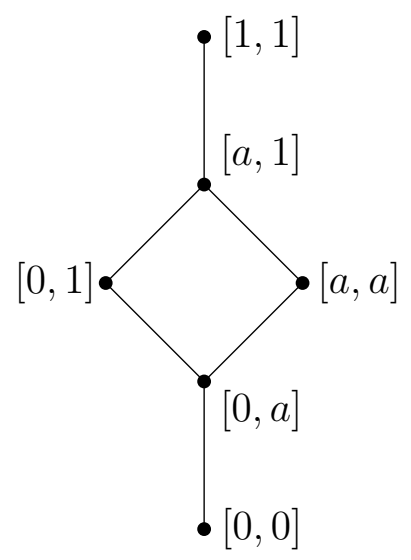

Fig. 4. The triangularization of a lattice with three elements. The considered IVRL corresponds to example 6.1.75(16) in [19].

ear iff $\nu x \sqcup \neg \nu x=1$ for all $x$ in $A$. Or equivalently, iff $x \sqcup \neg x=1$ for all $x$ in $E(\mathcal{A})$.

PROOF. Suppose first that $\mathcal{A}$ is prelinear. In particular, we know that ( $u \Rightarrow$ $\nu x) \sqcup(\nu x \Rightarrow u)=1$ for every $x$ in $A$. So $1=\nu 1=\nu(u \Rightarrow \nu x) \sqcup \nu(\nu x \Rightarrow$ $u)=((\nu u \Rightarrow \nu \nu x) \sqcap(\mu u \Rightarrow \mu \nu x)) \sqcup((\nu \nu x \Rightarrow \nu u) \sqcap(\mu \nu x \Rightarrow \mu u))=((0 \Rightarrow$ $\nu x) \sqcap(1 \Rightarrow \nu x)) \sqcup((\nu x \Rightarrow 0) \sqcap(\nu x \Rightarrow 1))=(1 \sqcap \nu x) \sqcup(\neg \nu x \sqcap 1)=\nu x \sqcup \neg \nu x$. Conversely, suppose $x \sqcup \neg x=1$ for all $x$ in $E(\mathcal{A})$. Then $(y \Rightarrow z) \sqcup(z \Rightarrow y)=1$ for all $y$ and $z$ in $E(\mathcal{A})$, as $z \sqcup \neg z \leq(y \Rightarrow z) \sqcup(z \Rightarrow y)$. So $\mathcal{A}$ is pseudo-prelinear and therefore distributive. Now we prove that $\mathcal{A}$ is prelinear as well. Remark first that $\neg x \sqcup y \leq x \Rightarrow y$, and $\nu y=\nu y \sqcup(\nu y \sqcap a)=(\nu y \sqcap \mu y) \sqcup(\nu y \sqcap a)$, for all $x, y$ and $a$ in $A$. So we have

$$
\begin{aligned}
1= & (1 \sqcap 1) \sqcup(1 \sqcap 1) \\
= & (((\nu y \sqcup \neg \mu x) \sqcup \neg \nu y) \sqcap((\nu y \sqcup \neg \mu x) \sqcup \mu x)) \sqcup \\
& (((\nu x \sqcup \neg \mu y) \sqcup \neg \nu x) \sqcap((\nu x \sqcup \neg \mu y) \sqcup \mu y)) \\
= & ((\nu y \sqcup \neg \mu x) \sqcup(\neg \nu y \sqcap \mu x)) \sqcup((\nu x \sqcup \neg \mu y) \sqcup(\neg \nu x \sqcap \mu y)) \\
= & \neg \mu x \sqcup(\neg \nu x \sqcap \mu y) \sqcup \nu y \sqcup \neg \mu y \sqcup(\neg \nu y \sqcap \mu x) \sqcup \nu x \\
= & (\neg \nu x \sqcap \neg \mu x) \sqcup(\neg \nu x \sqcap \mu y) \sqcup(\nu y \sqcap \neg \mu x) \sqcup(\nu y \sqcap \mu y) \sqcup \\
& (\neg \nu y \sqcap \neg \mu y) \sqcup(\neg \nu y \sqcap \mu x) \sqcup(\nu x \sqcap \neg \mu y) \sqcup(\nu x \sqcap \mu x) \\
= & ((\neg \nu x \sqcup \nu y) \sqcap(\neg \mu x \sqcup \mu y)) \sqcup((\neg \nu y \sqcup \nu x) \sqcap(\neg \mu y \sqcup \mu x)) \\
\leq & ((\nu x \Rightarrow \nu y) \sqcap(\mu x \Rightarrow \mu y)) \sqcup((\nu y \Rightarrow \nu x) \sqcap(\mu y \Rightarrow \mu x)) \\
= & \nu((x \Rightarrow y) \sqcup(y \Rightarrow x)) \\
\leq & (x \Rightarrow y) \sqcup(y \Rightarrow x),
\end{aligned}
$$


which concludes the proof.

Remark that the property $x \sqcup \neg x=1$ is called law of excluded middle (LEM) and that a residuated lattice satisfying LEM is always a Boolean algebra. Indeed, it is a Heyting algebra (and therefore distributive) because $x=x * 1=$ $x *(x \sqcup \neg x)=x * x \sqcup x * \neg x=x * x \sqcup 0=x * x$, for every $x$. Because a distributive lattice in which $x \sqcup \neg x=1$ and $x \sqcap \neg x=0$ holds is a Boolean algebra and because $*=\sqcap$, we find that the residuated lattice is indeed a Boolean algebra.

We now summarize the connections between properties on triangle algebras and properties on their diagonals in Table 1. We included the results from this section, and other equivalences that were proven in [28]. The table should be read like this: if the diagonal of a triangle algebra satisfies the given property (mentioned in the first column) and the value of $u * u$ is in the given range (mentioned on top of the column in question), then the triangle algebra satisfies the property at that place in the table. If also the converse holds (for the given value of $u * u$ ), then the property is underlined.

For example (on the fourth row of the table): if the diagonal of a triangle algebra is strong $\sqcup$-definable (in other words, an MV-algebra), then this triangle algebra is $\sqcup$-definable (no matter what the value of $u * u$ is). Conversely, if a triangle algebra is $\sqcup$-definable and $u * u=u$, then its diagonal is an MV-algebra.

Table 1

Properties on triangle algebras and their diagonals.

\begin{tabular}{c|ccc} 
diagonal & $u * u=0$ & $0<u * u<u$ & $u * u=u$ \\
\hline distributive & $\frac{\text { distributive }}{\mathrm{P}+\text { weak div }}$ & $\underline{\text { distributive }}$ & $\underline{\text { distributive }}$ \\
$\mathrm{P}+$ div & $\underline{\mathrm{PP}}$ & no inv & $\underline{\mathrm{PP}+\text { weak div }}$ \\
involution & $\sqcup$-def & $\sqcup$-def & no inv \\
MV-algebra & not Heyting & not Heyting & $\underline{\text { Heyting }}$ \\
Heyting & $\underline{\text { div }}$ & $\underline{\text { div }}$ & $\underline{\text { Heyting }}$ \\
Heyting $+\alpha$-LEM & $\underline{\mathrm{MV}}$ & $\underline{\mathrm{P}}$, not MV & $\underline{\mathrm{P}}$, not MV
\end{tabular}

In this table, $\mathrm{P}$ stands for prelinearity, PP for pseudo-prelinearity and LEM for law of excluded middle. The property $\alpha$-LEM means: for all $x$ on the diagonal, $x \sqcup \neg x \sqcup \alpha=1$, in which $\alpha=\mu(u * u)$. If $u * u=0$, this is the same as LEM; if $0<u * u$, this is weaker than LEM (for $u * u=u$ it is trivially satisfied). Remark that if 1 is $\sqcup$-irreducible (e.g., in linear residuated lattices), then $\alpha$ LEM is equivalent with LEM if $\alpha<1$. In this case the residuated lattice has 


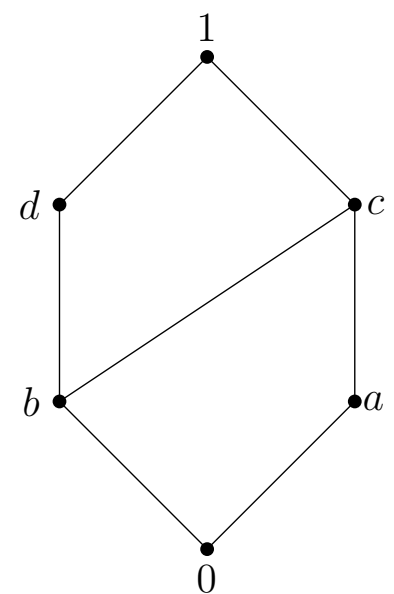

Fig. 5. LEM is not satisfied in the Heyting-algebra on this lattice since $c \sqcup \neg c=c \sqcup 0=c \neq 1$.

only two elements, as $\neg x=1$ iff $x=0$ because of the residuation principle. A residuated lattice with two elements is a Boolean algebra.

An example of a residuated lattice that does not satisfy LEM, but does satisfy $\alpha$-LEM for some $\alpha$ different from 1, is shown in Figure 5. Indeed, if we take the infimum in this lattice ${ }^{8}$ as $*$, then the negation $\neg$ is defined by $\neg 0=1, \neg a=d$, $\neg b=a, \neg c=0, \neg d=a$ and $\neg 1=0$. It is easy to verify that this residuated lattice satisfies $d$-LEM but not LEM. Moreover, it is also a Heyting-algebra. Therefore the triangle algebra corresponding with this residuated lattice and $\alpha=d$, will be divisible but not prelinear.

At this point it may be interesting to relate these results to some well-known implications and equivalences that hold in RLs.

- A residuated lattice is an MV-algebra iff it is divisible and the negation is an involution.

- A residuated lattice with involutive negation is $\sqcup$-definable iff it is weak divisible.

- If a residuated lattice is prelinear or divisible, then it is distributive.

- A divisible and $\sqcup$-definable residuated lattice, is prelinear.

- A prelinear residuated lattice is $\sqcup$-definable and weak divisible.

- A Heyting-algebra is divisible.

- A residuated lattice is a Boolean algebra iff it is a Heyting-algebra with involutive negation. It satisfies all of the properties mentioned here: distributivity, (weak) divisibility, (strong) $\sqcup$-definability and prelinearity.

\section{Conclusion and Future Work}

In this paper, we have introduced filters on triangle algebras to demonstrate that pseudo-prelinear triangle algebras are subdirect products of pseudo-linear

$\overline{8}$ This is example $6.1 .54(2)$ in [19]. 
triangle algebras. As a consequence, some properties hold for all pseudoprelinear triangle algebras if they hold for all pseudo-linear triangle algebras. We have given several examples of such properties. Another corollary is that the completeness theorem for Pseudo-prelinear Triangle Logic can be strenghtened.

It remains an open problem whether this completeness theorem can be reinforced even more, to standard completeness (i.e., w.r.t. the triangle algebras on $\mathcal{L}^{I}$ ), similarly as MTL being complete w.r.t. residuated lattices on $([0,1], \min , \max )$. This is a topic for further research.

\section{References}

[1] C.C. Chang, Algebraic analysis of many valued logics, Transactions of the American Mathematical Society 88(2), (1958), 467-490

[2] C.C. Chang, A new proof of the completeness of the Lukasiewicz axioms, Transactions of the American Mathematical Society 93(1), (1959), $74-80$

[3] R. Cignoli, F. Esteva, L. Godo, A. Torrens, Basic Fuzzy Logic is the Logic of Continuous t-norms and their Residua, Soft Computing 4, (2000), 106112

[4] C. Cornelis, G. Deschrijver, E.E. Kerre, Advances and challenges in interval-valued fuzzy logic, Fuzzy Sets and Systems 157(5), (2006), 622627

[5] G. Deschrijver and E.E. Kerre, Classes of Intuitionistic Fuzzy t-norms Satisfying the Residuation Principle, International Journal of Uncertainty, Fuzziness and Knowledge-Based Systems 11, (2003), 691-709

[6] R.P. Dilworth and M. Ward, Residuated Lattices, Trans. A.M.S. 45, (1939), 335-354

[7] F. Esteva, P. Garcia-Calvés, L. Godo, Enriched Interval Bilattices and Partial Many-Valued Logics: an Approach to Deal with Graded Truth and Imprecision, International Journal of Uncertainty, Fuzziness and Knowledge-Based Systems, Vol. 2(1), (1994), 37-54

[8] F. Esteva, L. Godo, Monoidal t-norm Based Logic: Towards a Logic for Left-Continuous t-norms, Fuzzy Sets and Systems 124, (2001), 271-288

[9] F. Esteva, L. Godo, A. Garcia-Cerdaña, On the Hierarchy of t-norm Based Residuated Fuzzy Logics, in: Beyond Two: Theory and Applications of Multiple Valued Logic (M. Fitting and E. Orlowska, eds.), Physica-Verlag, (2003), 251-272

[10] J. Font, A.J. Rodriguez, A. Torrens, Wajsberg algebras, Stochastica 8, (1984), 5-31 
[11] M. Gehrke, C. Walker, E. Walker, Some comments on interval-valued fuzzy sets, International Journal of Intelligent Systems 11, (1996), 751-759

[12] K. Gödel, Zum intuitionistischen Aussagenkalkül, Anzeiger der Akademie der Wissenschaften in Wien, (1932), 65-66

[13] S. Gottwald, Mathematical fuzzy logic as a tool for the treatment of vague information, Information Sciences 172, (2005), 41-71

[14] P. Hájek, Metamathematics of Fuzzy Logic, Trends in Logic-Studia Logica Library, Kluwer Academic Publishers, (1998)

[15] A. Heyting, Die formalen Regeln der intuitionistischen Logik, Sitzungsberichte der preuszischen Akademie der Wissenschaften, physikalischmathematische Klasse, (1930), 42-56 57-71 158-169 in three parts, Sitzungsber. preuss. Akad. Wiss.: 42-71, 158-169. English translation of Part I in Mancosu 1998: 311-327.

[16] U. Höhle, Commutative, Residuated l-monoids, in: Non-classical Logics and their Applications to Fuzzy Subsets: a Handbook of the Mathematical Foundations of Fuzzy Set Theory (U. Höhle and E.P. Klement, eds.), Kluwer Academic Publishers, (1995), 53-106

[17] E.V. Huntington, Sets of independent postulates for the algebra of logic, Trans. A.M.S. 5, (1904), 288-309

[18] S. Jenei, F. Montagna, A Proof of Standard Completeness for Esteva and Godo's Logic MTL, Studia Logica 70, (2002), 1-10

[19] P. Jipsen, Some lists of finite structures, at http://www1.chapman.edu/ jipsen/gap/rl.html,

[20] J. Łukasiewicz, A. Tarski, Untersuchungen über den Aussagenkalkül, Comptes Rendus de la Société des Sciences et des Lettres de Varsovie, (1930), $1-21$

[21] J.M. Mendel, Uncertain Rule-Based Fuzzy Logic Systems, Prentice Hall PTR, Upper Saddle River, New Jersey, (2001)

[22] J.M. Mendel, Advances in type-2 fuzzy sets and systems, Information Sciences 177, (2007), 84-110

[23] H. Rasiowa, R. Sikorski, The mathematics of metamathematics, Polska Akad. Nauk, (1963)

[24] B. Schweizer, A. Sklar, Statistical Matric Spaces, Pacific J. Math. 10, (1960), 313-334

[25] E. Turunen, Mathematics behind Fuzzy Logic, Physica-Verlag, (1999)

[26] B. Van Gasse, C. Cornelis, G. Deschrijver, E.E. Kerre, On the properties of a generalized class of t-norms in interval-valued fuzzy logics, New Mathematics and Natural Computation, Vol. 2 (No. 1), (2006), 29-42 
[27] B. Van Gasse, C. Cornelis, G. Deschrijver, E.E. Kerre, Triangle algebras: A formal logic approach to interval-valued residuated lattices, Fuzzy Sets and Systems 159, (2008), 1042-1060

[28] B. Van Gasse, C. Cornelis, G. Deschrijver, E.E. Kerre, A characterization of interval-valued residuated lattices, To appear in International Journal of Approximate Reasoning, doi: 10.1016/j.ijar.2008.04.006

[29] L.A. Zadeh, The concept of a linguistic variable and its application to approximate reasoning - I, Information Sciences 8, (1975), 199-249 